\title{
PAUL WILLEMS: LE DERNIER ÉCRIVAIN BELGE?
}

\begin{abstract}
Loriau Henri-François, Pantkowska Agnieszka, Paul Willems: le dernier écrivain belge? [Paul Willems: The last Belgian writer?]. Studia Romanica Posnaniensia, Adam Mickiewicz University Press, Poznań, vol. XXVIII: 2001, pp. 149-162, ISBN 83-232-1144-2, ISSN 0137-2475.

Paul Willems, Flemish writer and playwright, who wrote in French, can be considered along with Suzanne Lilar as one of the last Belgian writers in national understanding of this word.

This paper is a proposal of analysis of the last prose writing of Willems Le Pays noyé. As this work can be read as an allegory of Belgian history, attention is drawn to the problem of Belgian identity and its formation process.

Moreover the final remarks deal with the attitude of Willems to French language, reflected in his different dramatic writings.
\end{abstract}

Paradoxale qu'elle puisse paraître au premier abord, cette hypothèse initiale semble être confirmée par Paul Willems ${ }^{1}$ lui-même, lorsqu'il dit en 1978: «Suzanne Lilar, Paul Neuhuys, Guy Vaes, moi-même et quelques autres sommes les derniers représentants d'une race en voie de disparition» ${ }^{2}$.

La race en voie de disparition est celle de l'écrivain belge. Il ne s'agit pas de l'écrivain en tant que tel, de son statut ni de son métier; c'est le qualificatif: belge

I Paul Willems né à Edegem en 1912. Fils de la romancière francophone Marie Gevers et de Franz Willems, parent de Jan-Frans Willems, le «père du mouvement flamand». Elevé dans la culture française, comme beaucoup d'enfants de la bourgcoisie flamande de l'époque. Docteur en droit, il commence sa carrière littérairc par des romans: Tout est réel ici (1941), Blessures (1945); connu surtout pour ses pièces de theatre: Le bon vin de Monsieur Nuche (1949), La plage aux anguilles (1959), Warna (1962), La ville à voile (1967). Il est mort en 1997. Pour plus d'information concernant l'homme et son ocuvre voir aussi Le Carnet et les instants, N" 101, Le Monde de Paul Willems. Textes, études, documents rassemblés par P. Emond, H. Ronse et F. van de Kerckhove, Labor, Bruxclles 1984, p. 241 (Coll. Archives du futur), M. Quaghebeur, Lettres belges entre absence et magie, ed. Labor, Bruxclles 1990, pp. 221-24I, M. Quaghcbeur, Alphabet des lettres belges de langue française, Association pour la promotion des Lettres belges de langue française, Bruxelles 1982, pp. 159-163, p. 304.

2 Le monde de Paul Willems, op. cit., p. 230. 
qui pose problème. En effet, le terme de littérature belge renvoie au concept d'une littérature nationale. Son modèle, forgé dès 1830 , est basé sur le principe biculturel (fusion de la culture française et de la culture flamande) et unilinguiste: la langue d'expression littéraire est uniquement la langue française. Ce modèle, restant en parfait accord avec le concept de «l'âme belge» est introduit par le texte fondateur de la littérature belge: La légende d'Ulenspiegel de Charles De Coster et il a vite trouvé des illustrateurs de talent: Rodenbach, Verhaeren et Maeterlinck grâce à qui la littérature belge nationale se hisse au rang des grandes littératures mondiales. Toutefois, parallèlement aux revendications séparatistes flamandes, la réalité politique de la Belgique change. Secoué par la guerre linguistique le rêve unioniste belge s'évanouit et avec lui la littérature nationale qui cède aujourd'hui la place à la littérature néerlandophone et à celle francophone de Belgique.

Paul Willems, Flamand francophone, est donc bel et bien l'un des derniers grands écrivains belges de son temps. La conscience de l'inévitable cassure, la nostalgie de la Belgique unie, le bilan historique de la Belgique ainsi qu'un message ultime: tels sont les thèmes qu'on retrouve dans son dernier texte en prose édité en 1990 et intitulé Le pays noyé . $^{3}$

Ce récit constitue une manière d'allégorie dramatique qui rappelle singulièrement l'histoire de la Belgique. Ainsi que cette histoire se divise, classiquement, en trois grandes phases; de même dans ce récit de Willems on peut distinguer trois étapes relatives à la construction d'une identité nationale, à la confrontation de deux tendances opposées et à l'éclatement du pays".

\section{LE PAYS NOYÉ: LA BELGIQUE?}

Le pays noyé, qui se nomme Aquélone, est un pays, une ville plus exactement, situé au bord de l'estuaire de l'Escaut et dirigé par un bon et paisible Empereur. La référence à l'Escaut est significative. C'est un fleuve emblématique belge, au même titre que la Vistule en Pologne, source d’inspiration littéraire de toute une pléiade d'écrivains belges, parmi lesquels Verhaeren et Gevers. Et enfin ce fleuve qui prend sa source en France et qui se jette dans la Mer du Nord, constitue un véritable trait d'union entre les territoires flamands et francophones, germains et latins. Une autre référence à la réalité belge: si l'Escaut est le fleuve-emblème du pays, la bière, unique boisson à Aquélone, en est le symbole aussi étant considérée comme boisson nationale.

\footnotetext{
${ }^{3}$ P. Willems, Le pays noyé, Fata Morgana, s. 1, 1990, 85 p.

${ }^{4}$ En ce qui concernc les données concernant ces trois étapes, cf. J.-M. K l in ke nberg, La production littéraire en Belgique francophone. Esquisse d'une sociologie historique, dans Littérature, $n^{\circ} 44$ L'insittution littéraire 11,1981 , p. 33-50. M. Quag h e bcur, Belgique. La première des littératures francophones non françaises, Luxembourg, Association des Professeurs de Français du Grand-Duché de Luxembourg, 1991 (Coll. Les Cahiers Pédagogiques).
} 


\section{I. L'ÈRE DE L'INEFFABLE: LA CONSTRUCTION D'UNE IDENTITÉ}

Aquélone est un pays de bonheur et de prospérité qui se suffit à lui-même. A Aquélone, la vie se passe sereinement et les relations humaines s'épanouissent de manière originale: ni possession ni jalousie dans les relations amoureuses qui se font selon le bon vouloir des filles-d'eau, «dispensant» leurs grâces sans engagement. Entouré d'une telle félicité, l'Empereur avait même abrogé toutes les lois et déposé sa couronne. Cet aspect édénique est d'avantage accentué par le sentiment de sécurité totale assuré par les paralumières, sorte d'écrans géants que les Aquéloniens ont construits pour se protéger des rayons du soleil. Cependant ces paralumières, joliment décorés, limitent le jugement. le regard objectif des habitants en leur renvoyant les perspectives et coupant l'horizon. Cette «ère de l'ineffable» (p. 8) n'est pas sans rappeler le bonheur unitaire dans lequel vit la nation indépendante en 1830. La bourgeoisie francophone ou francisée du XIX ${ }^{\mathrm{e}}$ siècle développe l'idée d'une âme belge qui serait la synthèse de l'esprit germanique et de la culture latine; c'est le projet d'un génie national qui s'exprimerait en français. Une part des oeuvres historiques et littéraires contribue à la création de cette identité montée de toutes pièces. En parallèle, à partir de 1880 émergent de grands auteurs flamands s'exprimant en français qui vont largement contribuer au processus de légitimation internationale du pays (songeons aux grands symbolistes, avec notamment Maeterlinck qui reçoit le prix Nobel de littérature en 1911 pour son ouvrage Monna Vanna) ${ }^{5}$. Malgré les premières revendications linguistiques flamandes qui conduisent aux premières reconnaissances linguistiques dès les années 1870 en Belgique, on observe une convergence de forces qui tendent à la construction d'un Etat et à son rayonnement. Sans doute cette première phase constitue-t-elle pour la nation l'ère de l'ineffable.

\subsection{LE DUEL DES DIGNES: LA FIN D'UN RÊVE}

Toutefois le bonheur indicible des habitants d'Aquélone est troublé par une fille-d'eau, Althéna, qui bravant la règle de non-engagement, décide de s'attacher à un seul homme, à Herk. Cette transgression à un rituel aquélonien constitue une brèche dans la perfection du système exposé dès lors à un danger. Effectivement, le soir même, une grande tempête survient et laisse des déchirures dans les paralumières, que personne ne songe à réparer: «Peut-être se rendait-on compte qu'un ciel ravaudé serait pire qu'une déchirure avouée?» (p. 14). Progressivement, le trouble croît dans la population qui pourtant demeure inactive: «On se sentait menacé. Par qui? Par quoi? En chacun, une autre déchirure s'ouvrait sur un gouffre. Combien de temps pourrait-on se le cacher?» (p. 14).

${ }^{5}$ A propos de ces autcurs tlamands francophones, cf. notamment Ch. Berg, D'un pays noyé: la litterature francophone contemporaine en Flandre, dans Septentrion. Revue de culture néerlandaise, 2 le annéc, $\mathrm{n}^{\circ} 4,1992$, p. 49-52 ou, du même autcur: La Belgique romane et sa Flandre, in Littératures belges de langue française, Ed. Lc Cri, Bruxelles 2000, pp. 389-407. 
La situation analogue se produit en Belgique, ou la tempête séparatiste brise la cloche unioniste. Les paralumières de la Belgique, c'est cet étonnant fantasme d'une identité germano-latine ayant pour seule expression le français. Car en même temps, à la fin du XIX ${ }^{\mathfrak{e}}$ siècle, les revendications flamandes ont abouti à l'emploi du flamand pour une part de la justice, pour l'enseignement secondaire, pour une part de l'enseignement universitaire et, en 1898, au bilinguisme dans la publication des textes officiels. Le rêve du pays biculturel, mais unilinguiste rejoint d'autres mythes de grandeur de Belgique, car les déchirures apparaissent dans l'Etat, le menacent, sans que les responsables s'en rendent compte.

A Aquélone, afin de calmer les esprits, l'Empereur croit bon de faire revivre une ancienne coutume: le Duel des Dignes qui rappelle le duel légendaire entre Routroux et Valdo. Deux hommes devaient s'ensabler jusqu'aux genoux sur un banc de sable de l'estuaire, à marée basse. L'eau montante les emprisonnait et ils devaient alors se frapper à coups de gourdins jusqu'à ce que l'un d'eux soit tué ou se noie, assommé. Persuadé de l'influence bénéfique du duel sur les esprits en ébullition, «l'Empereur ne se rendait pas compte qu'il allait déclencher en Aquélone les mécanismes de l'angoisse et de la cruauté» (p. 19). D'autant plus que pour ce duel, deux frères sont désignés: Herk, choisi auparavant par Althéna, et Liou ${ }^{6}$, son favori actuel.

C'est Herk qui gagne le duel. La marée redescendue, Herk libère la dépouille de Liou, mais au lieu de la laisser aller dans le courant, il la ramène à Aquélone. A la vue du cadavre, l'Empereur et le peuple sont choqués. Et le soir même, une autre grande tempête s'abat sur Aquélone. Pour conclure cet épisode, Willems écrit: «Le destin d'un pays se trouve ainsi parfois en suspens. Moment terrifiant, quand on découvre qu'on s'est engagé dans un chemin qui mène au gouffre et qu'il est trop tard pour reculer» (p. 34). De ce duel, tous attendaient le retour à la sérénité; c'est finalement l'inverse qui se produira...

En Belgique, Flamands et Wallons sont belges, fils d'une même patrie ils sont donc frères. Cela ne les empêchera pas de s'opposer dans un véritable duel.

Dans le récit de Willems, les allusions semblent être nombreuses et équivoques. L'infidélité d'Althéna, par exemple, pourrait rappeler l'infidélité à la patrie, consommée de part et d'autre, mais aussi l'infidélité de la langue, le français ne suffisant plus aux deux parties.

L'impuissance des deux frères à empếcher l'issue fatale rappelle curieusement l'impuissance des citoyens à empêcher la désagrégation nationale. Liou et Herk n'ont pas choisi ce combat; à partir d'une certaine époque, le combat communautaire en Belgique n'est plus le fait de la masse mais de ses élites et d'une minorité agissante, distorsions des volontés et impuissance à éviter l'issue.

Liou pourrait encore préfigurer cette Belgique première, synthèse fantasmatique de deux cultures, tandis que Herk symboliserait cette autre Belgique, encore unie bien que divisée, mais dont l'avenir n'est guère plus brillant.

${ }^{6}$ Il est possible de voir dans le choix onomastique de ces personnages les références à la langue flamande Herk, et à la langue française ou Liou serait une variante de Louis. 
Politiquement, la Belgique devient bilingue en 1932. Mais le véritable tournant se situe plus tôt, lors du cataclysme de 14-18. Après une brève période d'entente nationale, au sortir de la guerre, l'Etat commence son inexorable décomposition. C'est la fin des rêves de génie national à deux têtes, l'identité synthétique germano-latine, l'inanité du projet syncrétiste officiel est découverte.

La vision de la Belgique, pont entre les cultures, s'estompe! En politique comme en littérature, la rupture est de plus en plus profonde et irréversible. Les littérateurs francophones du pays vont alors peu à peu adopter deux attitudes distinctes: soit ils vont opérer un repli vers la «mère-patrie linguistique», gardienne des valeurs culturelles francophones? ${ }^{7}$, soit ils vont observer une attitude de dénégation de l'appartenance belge ${ }^{8}$.

\subsection{UNE VILLE-EN-RUINE: LA RUINE D'UNE NATION}

Enterré dans la cabane d'Althéna, «le corps pourrissant de Liou allait grandir comme un chancre» (p. 48). Aquélone commence sa lente désagrégation; la ville tombe peu à peu en ruines, mais, précise Willems, dans ce pays on ne disait pas «détériorations» mais «changements» (p. 57). Tout comme en Belgique, ou le chancre se développe aussi et la ruine de la nation n'est pas ponctuée non plus de détériorations, mais des réformes.

Les fidèles d'Althéna, devenue manière de prêtresse, sont de plus en plus nombreux et se font chaque jour plus violents. Ils mettent graduellement la ville à sac et provoquent la terreur au sein d'une population qui ne comprend pas mais ne réagit pas non plus. A propos de ces fidèles, l'Empereur dira: «Je ne sais pas ce qu'ils cherchent, ni où ils cherchent. Je ne sais même pas quelle est la question. Ils ont l'air de ne pas pouvoir se passer de. Se passer de souffrir» (p. 74). Destructions et répressions sont terribles en ville, et bientôt «Aquélone n'est plus une ville-en-songe mais une ville-en-ruine» (p. 68).

Poussé à bout par Althéna, Herk s'en va finalement mourir de froid et de mauvais traitements sur un tas d'immondices. Sa dépouille sera enfermée auprès de celle de son frère Liou, main dans la main, dans un gigantesque mausolée.

L'Empereur décide alors de quitter la ville et de se laisser mourir. L'ancienne Aquélone est définitivement morte; Althéna sera l'Impératrice de la nouvelle et fera

${ }^{7}$ Ce sera une forme de repli vers la France. Songeons à Franz Hellens et au Manifeste du Groupe du Lundi rédige par ces auteurs «rançais-bclges»; songcons également à quelqu ‘u comme Charles Plisnier, internationaliste en 19/9, prix Goncourt en 1937 pour Fatux-passeports et qui aura des tendances rattachistes lors de la seconde guerre mondiale.

${ }^{8}$ Certains s'expatrient, comme Michaux, d'autres, comme les surréalistes, ont des prises de position internationalistes, d'autres encore cherchent des lieux irréels pour leur littérature, hors de cette réalité qui explose, comme Ghelderode ou Crommelynck, d'autres enfin se lancent dans le fantastique ou la "paralittérature», comme Jean Ray, Simenon ou Hergé... 
régner la fausse grandeur, l'optimisme de commande, la violence et l'éradication systématique de la mémoire.

L'analogie avec le devenir de la Belgique est ici aussi frappante. Politiquement, après bien des vicissitudes, l'Etat unitaire est officiellement divisé en 1992: la Belgique devient un Etat Fédéral composé de Régions et de Communautés. Après avoir été une nation-en-songe, la Belgique est devenue une nation-en-ruine!

En littérature, la situation n'est guère plus stable. En parallèle des luttes linguistiques et communautaires qui divisent le pays, la Belgique tombe dans un marasme économique qui touche surtout la partie francophone'; et en même temps que décline le concept de nation se développe le concept de francophonie et la réalité européenne supranationale. Sur ce sol qui vient à manquer, où tout s'écroule, les littérateurs francophones de Belgique s'inscrivent dans une réalité qui s'effrite et tombent dans un profond malaise.

Ce malaise s'est entre autre manifesté par une réaction qu'il a provoquée chez certains auteurs. Dans les années septante apparaît le concept de «belgitude». Construit sur le concept de négritude, il correspond à un compromis entre diverses attitudes. Il est à la fois la marque de l'acceptation du fait d'être né quelque part, en Belgique, d'en tenir compte, d'y vivre et de s'y battre. Il est en outre l'indice d'un double refus: celui de la dénégation permanente de son identité belge et celui de l'exacerbation de son appartenance au point de réduire sa définition à son origine, au risque d'aboutir à un quelconque nationalisme. La belgitude, c'est donc la volonté d'une identité poreuse cosmopolite, ouverte sur l'Europe et sur le monde et en échange permanent avec la France.

Ce malaise des auteurs francophones belges et cette recherche sur leur identité correspondent à la troisième phase de la littérature belge de langue française. Cette phase ouvre à l'interrogation sur le manque qui touche à l'être et à la langue. A une certaine époque, pas si lointaine, il était difficile pour certains de nos auteurs d'être francophones non français dans un contexte à ce point instable et dans une langue «autre».

Institutionnellement, ce problème est bien illustré par l'ambiguîté de cette dénomination de «Communauté Française de Belgique», avec ce qualificatif qui signifie l'appartenance à la fois à une langue et au territoire de la République. Cette formulation indique clairement «l'absence de terme pour désigner le peuple roman du Nord de l'Hexagone»" ${ }^{10}$.

Pas ou plus de pays à soi, pas de langue bien à soi, ou tout au moins pas reconnue comme telle, tous ces malaises aboutissent à de fortes réactions dans les ouuvres: la

${ }^{9}$ En 1960 , la Belgique perd sa très belle colonie, assise économique du pays, surtout de la partie francophone. Dans la même période survient progressivement le déclin des charbonnages et de la grande sidérurgie lourde du Sud du pays. Peu à peu par contre le Nord commence à monter un système économique viable, mieux adapté aux réalités du temps.

${ }^{10}$ M. Quaghebeur, Belgique, op. cit., p. 30. 
difficulté de dire «je» pour parler d'eux-mêmes", la transposition de l'instabilité ambiante et des dérives de notre époque ${ }^{12}$, la recherche d'une autre forme d'écriture ${ }^{13}$, ou encore le retour d'un nouvel éloignement par rapport au réel, ainsi que l'écrit Willems dans ses jeux de superposition du rêve et du réel, dans ses constructions en miroir, avec ses personnages anéantis par le contact au réel, par sa recherche permanente d'un passé révolu...

Willems donc répond à certaines constantes de son «époque littéraire belge», de même qu'il écrit et use d'un langage marqué par son contexte.

\section{PAUL WILLEMS: UN TRAVAIL ET UNE RÉFLEXION SUR LA LANGUE}

En 1893, Max Elskamp écrivait: «Je regrette de ne pas savoir le flamand. C'était la langue qu'il m'aurait fallu, puisque le belge n'existe point ... En d'autres termes, je ne puis travailler car je ne suis plus sûr de savoir une langue! Quelle bonne chose ce serait d'être un pays à soi, fût-ce la Belgique si ça existait!» ${ }^{14}$

Trente-cinq ans plus tard, Willems écrivait, au sortir de l'adolescence: Je ne comprends pas le français, je connais mal le flamand, je n'ai pas de langue» ${ }^{15}$.

Ce problème du flamand francophone n'est donc pas nouveau; Willems, pour sa part, ressent un double malaise: le premier par rapport au français de France, le second par rapport à son milieu flamand.

\subsection{UN DOUBLE MALAISE: \\ UN AUTEUR FRANCOPHONE NON FRANÇAIS DE FLANDRE}

Un auteur francophone non Français de Flandre, comme Willems, est ainsi constamment «entre-les-deux»: il est Flamand, mais la langue flamande se refuse à lui, il est francophone, mais souffre de ne pas la posséder pleinement.

Cependant, ce double malaise n'a pas empêché Willems d'écrire; au contraire, il considère que ses relations particulières à la langue et à son milieu sont à la source de sa création et des spécificités de son écriture.

11 A l'image de Mertens qui ne parle de son pays que par l'intermédiaire d'un tiers, qu'il s'agisse d'un exilć chilien dans «Terre-d'asile» ou d'un poète allemand dans «Les Eblouissements».

${ }^{12}$ Comme Louvet qui intègre dans son theảtre un univers dans lequel se dérobe le réel, comme Kalisky qui rappelle encore les traumatismes provoqués par le nazisme.

13 Ainsi que le rappellent notamment les logogrammes de Dotremont.

${ }^{14}$ Cité après P. Willems, le monde de Paul Willenss, op. cit., p. 229.

${ }^{15} \mathrm{P}$. W illems, L'auteur dramatique flamand de langue française, dans Etudes de littérature française de Belgique offertes à Joseph Hanse pour son 75 e anniversaire, éd. Jacques Antoinc, Bruxelles 1978, p. 31. 
La maîtrise approximative de la langue française l'a en quelque sorte soutenu dans sa démarche d'écrivain: selon lui, l'écrivain doit trouver sa propre langue et non se contenter de retranscrire la langue usuelle ou scolaire. Willems, dans ses œuvres, cherche à traduire ses impressions en images ou en mots, sans inscrire sa langue dans un système de pensée prédéfinie. Il s'agit de relier le plus étroitement le monde et ce qu'il écrit, en évitant l'écran de la langue. Dans ses textes, il propose des mots qu'il a vécus et non pensés. Parmi ses néologismes, il faut citer, à titre d'exemple:

- Bientôt les jardins tuliperont ${ }^{16}$.

- Atteint d'anthipatite virale pour mes concitoyens, je me suis expatriét ${ }^{17}$.

- Elle jouit de jambes fuselées et d'une poitrine boinbelée ${ }^{18}$.

- Exactement, kif kif, identostructes ${ }^{19}$.

- Herman, pâle bureaucrate, découvre un jour sa triste nature en se voyant dans un miroir: Je suis un homme qui ne parle jamais à haute voix tout seul, un honme d'avenir bancaire. Un fondé de pouvoir, un fondu de couloir. (tristement) un fond de tiroir ${ }^{20}$.

- Pour nommer une paire de pantoufles, chargée de l'affection que l'on montre aux objets longuement utilisés, d'aspect oriental, fatiguée par l'âge, il emploie un terme bâtard issu des mots "pantoufle» et «sloef» (équivalent en flamand) qui lui semble rendre ce qu'il ressentait: il les nomme «luffelines» ${ }^{21}$.

Les inventions verbales chères à Willems ne tombent pas dans la facilité des jeux de mots abstraits ou du calembour gratuit. Conscient de cet écueil, il affirme que le travail d'invention doit être guidé par une forme d'inspiration inconsciente, elle-même issue des personnages ou de la situation. Il voudrait que le lecteur / spectateur ait cette sensation que ses textes sont faits sur mesure et qu'il puisse concevoir, en les découvrant en même temps que les décors et les costumes, l'unité du texte et du destin de la pièce ${ }^{22}$. Il ne faudrait en outre pas confondre cette intrusion de l'inconscient dans la création de Willems avec un quelconque avatar de l'écriture automatique chère aux surréalistes. Cette confusion est en effet impossible car, à ce qu'il qualifie d'inspiration, il oppose un tri sévère au terme duquel il ne conserve que les mots qu'il considère comme les plus heureux et les plus adéquats.

${ }^{16}$ P. Willems, La Chronique du Cygne, Plon, Paris 1949.

${ }^{17}$ P. Will em s, Nuit avec ombres en couleurs, Bruxelles, 1983, p. 13 (Coll. Textes pour Didascalies $\left.n^{0} 6\right)$.

${ }^{18}$ P. Willems, Nuit [...], op. cit., p. 15.

19 P. Willems, La Ville à voile, Labor, Bruxelles 1989, p. 36 (Coll. Espace Nord n" 55).

${ }^{20}$ P. Willem s, Il pleut dans ma maison, le Rideau de Bruxclles, Bruxclles 1976, p. 60 (Les Cahiers du Rideau $\mathrm{n}^{\circ} 3$ ).

${ }^{21}$ Le Monde de Paul Willems. Textes, études, documents rassemblés par Paul Emond, Henri Ronse et Fabrice van de Kerckhove, Labor, Bruxelles 1984, p. 24 I (Coll. Archives du futur).

22 Id., p. 242. 
Depuis l'éclatement des conventions, l'auteur contemporain n'a plus recours à une quelconque solution formelle. L'oeuvre contemporaine s'inspire de la réalité et se plie à ses propres lois. On comprend dès lors le problème qui se pose à ce Flamand d'expression française, qui de plus a pour objectif, dans son écriture, de coller le plus étroitement à son monde: comment se référer à une réalité qui s'exprime dans une autre langue, $s$ 'il veut éviter le mensonge et l'approximation? Et ce problème se pose également pour l'écrivain belge en général: comment écrire une réalité instable qui s'exprime en plusieurs langues? Sans doute cette question ne se pose-t-elle pas pour l'auteur français, allemand ou anglais qui pourra choisir de nommer son personnage Monsieur Dupont, Herr Müller ou Mister Smith. Ce seul nom situe socialement le personnage et lui commande son langage. En Belgique, le recours unique serait Monsieur Beulemans ${ }^{23}$, qui s'exprime dans une langue faite de flamand et de français. Mais cette seule référence ne suffit pas à rendre compte de cette réalité multiple que constitue la Belgique.

Or, puisque les problèmes essentiels au théâtre concernent le temps, le lieu et la langue (forme de l'action selon Willems), l'auteur belge, ne disposant pas d'un langage de référence, est en même temps privé de temps et de lieu. En effet, comment faire dire à un personnage qui habite Anvers «il pleut» alors que nous savons qu'en réalité il dit «het regent»?"24.

A ce problème essentiel, les solutions mises en place par les auteurs belges sont diverses, mais se rejoignent toutes sur une idée commune: la nécessaire transposition de la langue, du lieu, du temps, en tout ou en partie. Si Maeterlinck situe ses pièces dans des châteaux ou des forêts intemporelles et imaginaires, que Crommelynck gomme toute référence au réel dans son espace théâtral ou Ghelderode renvoie plusieurs fois à une Flandre mythique, rêvée dans les profondeurs du XVI ${ }^{\mathrm{e}}$ siècle. Willems propose une solution «autre».

\subsection{LA SOLUTION DE WILLEMS: UN AUTRE LIEU, UNE AUTRE LANGUE}

Willems également a choisi de concevoir l'espace théâtral en fonction de son malaise. Ainsi, le lieu de l'action dans ses pièces est soit vague, soit confiné, et parfois les deux à la fois. Les didascalies de localisation en début de pièce sont éloquentes en sont la preuve. Dans Nuit avec ombres en couleurs il est précisé que «la scène est

${ }^{23}$ Personnage de la celc̀bre pièce de Fonson et Wichcler, Le mariage de Mademoiselle Beulemens. Beulemans est le stéréotype d'un Bruxellois s'exprimant en kaekebrocck, mais par ce fait-là il ne couvre qu'une partic de la population belge.

${ }^{24}$ Les origines et les implications de ce double malaise sont presentées par Willems lui-même dans: $\mathrm{P}$. Willems, $A$ la recherche d'un langage, dans Marginales. Revue bimestrielle des idées et des lettres, n" I12-113, mai 1967, p. 105-106. 
devant une palissade. Trois portes plus les entrées de côtém ${ }^{25}$; dans Le marché des petites heures: «Un bidonville. Les murs et les toits des petites maisons sont faits de caisses et de boîtes à conserves dépliées. On lit sur les murs: pommes, ananas [...] Les boîtes à conserves [...] sont de couleur vive et leur combinaison forme des calligrammes ou des «collages» d'un tableau dada ${ }^{26}$, tandis que dans La Vita Breve: «La scène est à bord d'un navire à la fin du XIX ${ }^{\mathrm{e}}$ siècle» ${ }^{27}$.

Willems ne se limite pourtant pas seulement à gommer toute référence précise au lieu et au temps de l'action. Il affirme que toute son ceuvre est issue de son arrièrepays», de son monde du dedans, en d'autres termes de sa mémoire profonde ${ }^{28}$. C'est ce qui lui permet de parler de mots vécus, c'est ce qui justifie sa recherche d'adéquation entre ses impressions et son écriture. Pour la transcription de cette réalité digérée et rendue, mystérieusement additionnée d'une charge affective et très probablement modifiée, il situe l'intrigue dans un espace autre, irréel, bien que concret, comme c'est le cas de La Ville à Voile ${ }^{29}$.

La déréalisation de l'espace ${ }^{30} \mathrm{~s}$ 'accompagne de l'altération du langage des personnages: Anne-Marie (vendue par son fiancé, Dile, à Josty qui la veut pour épouse parce que celle-ci ressemble à s'y méprendre au mannequin Fenêtre), aveuglée par son amour, répond toujours «à côté» de la question; les époux Roi constituent une caricature de la petite bourgeoisie étriquée, instruite mais limitée: Monsieur Roi possède un riche vocabulaire et l'étale largement, Madame Roi, en revanche, a la syntaxe courte et développe une phraséologie incomplète ${ }^{31}$. Enfin Agréable, le domestique des Roi, répète toujours trois fois les formules de politesse sous l'injonction de sa patronne.

Le questionnement du langage est également visible dans d'autres pièces de Willems. Dans Nuit avec ombres en couleurs, Vincent, jeune homme traumatisé par la mort de sa fiancée et surtout par la jalousie incestueuse et tyrannique de sa soeur Josée, dévoile de sérieux troubles du langage. Il comprend mal et s'exprime confu-

${ }^{25}$ P. Willems, Nuit [...], op. cit., p. 8.

${ }^{26}$ P. Willem s, Le Marche des petites heures, Bruxelles 1983, p. 65 (Coll. Texte's pour Didascalies $\left.n^{\circ} 6\right)$

${ }^{27}$ P. Willems, La Vita Breve, Labor, Bruxelles 1989, p. 150 (Coll. Espace Nord $\mathrm{n}^{0}$ 55).

${ }^{28}$ A ce propos, cf. P. Willems, Un arrière-pays. Réveries sur la création littéraire, Louvain-la-Neuve, Presses universitaircs de Louvain UCL, 1989 (Coll. (haire de poétique n"3).

${ }^{29}$ P. Willems, La Ville à voile, Bruxelles, Labor, 1989, p. 13-148 (Coll. Espace Nord n" 55).

${ }^{30}$ Même s'il précise que l'action se déroule à Anvers, ce glissement vers une forme d'aillcurs se traduit dans la conception de l'espace théâtral de la piècc: il est confinc au ubric-ì-brac" des époux Roi et il est décoré de manière presque surrealiste, contextualisant ainsi l'intrigue dans un milieu bien peu récl.

${ }^{31}$ Monsicur Roi (à Anne-Maric): Qu'as-fu fait de deux à quatre heures? Anne-Marie: Comme chaque année, le dix novembre, il y a une rempêre.

[...] Madame Roi (alias Paysage; à Monsicur Roi): Celte façon de répondre à côté! Elle te ressemblè. Monsieur Roi: Non, à tor.

Madame Roi: Moi, je crois que.

P. Willems, La Ville (...), p. 22-23 (cf. également la preface de J.-M. Picmme, p. 7-12). 
sément: les phrases viennent avec difficultés et les mots se brisent ${ }^{32}$. En manière de contrepoint aux hésitations langagières de Vincent apparaît le Chat Astrophe, personnage zoomorphe au masque de félin, sorte de narrateur omniscient, voix off et Monsieur Loyal, tout en même temps: celui-ci, à l'inverse, s'exprime dans une langue fluide, mais ses propos restent cependant surprenants et contribuent à la construction à nouveau d'un cadre relativement loufoque pour la mise en scène de personnages au destin malheureux ${ }^{33}$.

Dans Elle disait dormir pour mourir, Hélée, depuis le début de la guerre, est seule à l'abri dans la Maison-des-marais. Son pěre est parti au combat et sa mère, psychologiquement faible, a disparu à la recherche de celui-ci. Hélée a dix-huit ans; depuis sept ans elle a pour seule compagnie les bruits de la nature et un Larousse de poche en guise d'interlocuteur. Soudain arrive un soldat et dès leurs premiers échanges, la jeune fille dévoile un langage bien particulier: «éduquée» par le dictionnaire, elle le connaît par coeur et donne aux mots une signification toute neuve, libre du poids de l'usage. Ainsi dit-elle «bonheur» pour «vent», «oiseau» pour «souvenir», «saison» pour «avenir» ou encore «dormir» pour «mourir»" ${ }^{34}$. Au terme de ces longues années de solitude, le langage n'est plus pour elle un outil de communication mais le dernier

32 Vincent: Moi je ne lui at rien donne. Je ne lui ai pas donne mon adieu de vivant. Je ne lui ai rien. dit, Bella. conme c'est etrange de se remettre à parler. Après un an de silence. Je ne pounais pas. ça fait mal et pourtant c'est une blessure qui se rouvre et saigne. A l'instizut, je ne pouvais pas. Ma soeur me frappait pour que je crie. Elle disait que si je criais, après je pourrais paraler. Mais en moi, c'létait du sable sec qui buvait les, avant qul'elles n'arlivent, je veux dire avant qul'elles n'arlivent, qu'elles n'arlivent avant qul'elles. Je vleux dite avlant qules. (Silence. Il sourit). P. Will cm s, Nuit l...l, op. cit., p. 28.

${ }^{33}$ Chat: Fin de la presentation de Josée et de son frère Vincent. C'était il y a un an. (II annonce). Chronique du monde pendant un an. Lés nuages ont glissé, sans bruit, de l'ouest à l'est, glissé sams bruat. Il y a eu l'automne, l'automne de l'arbre et puis l'hiver, l'hiver de l'arbre. Et maintenant les nouvelles feuilles poussent. J'ai vu passer très haut dans le ciel "l'oiseau Pi-Hi, qui n'a qu'une aile et ne vole que par couple». Signalons pour être complet que dans le monde trente millions d'enfants sont morts de faim celte année. Mais il y a encore beaucoup, beaucoup d'enfants en vie, dont trente millions au moins mourront l'amuée prochaine. Voilà pour une année du monde. P. Willems, Nuit [...l, op. cit., p. 11.

Cette replique permet de souligner au passage unc autre particularité des textes de Willems. Régulièrement, l'humour et le bcau sont étroitement reliés dans les propos avec la cruauté ct l'horreur; le passage de l'un à l'autre se fait sans transition, ce qui ne manque pas de produire des effets sur le public ct de crécr une atmosphere un pcu particulière pour certaines de ses pièces.

${ }^{34}$ Hélée (au soldat): "Titoyer.» Attends. (Elle cherche dans sa mémoire.) «Tutoyer.» Ah oni! ça vient après tutoiement, action de tutoyer, après tutoyer il y a (elle récite à toutc vitesse, très ficre, comme un pianiste doué qui fait des arpèges prestissimo): tutu, tuyau, tuyatage, tuyauter, tuyauterie, tuyère, tumpan, tvpanique, ef type. Type, c'est: "personne originale», «un type curieux». Je crois que tu es un type curieux. (Intriguéc:) Qu'est-ce que tu tiens là dans la main?

Le soldat (il crie): in re-vol-ver!

Hélée (ravie): Revolver! "Arme à feu portative de petite taille dont l'approvisiomnement est automarique».

P. Willems, Elle disait dornir pour mourir, le RdB, 3c éd. Bruxelles 1994, p. 34 (Les Cahiers du Rideau n" 16 ). 
lien avec le monde des vivants. D'une certaine façon, elle possède le vrai parler, celui de l'âme des choses: pour elle. les mots ne signifient pas leur apparence mais existent en eux-mêmes ${ }^{35}$.

Dans La Vita Breve, embarqués sur un bateau, les personnages sont tous les acteurs d'un drame guidé par la jalousie et les obsessions refoulées. Le Vélicouseur, victime de l'amour incestueux de sa mère, jaloux lui-même jusqu'au dérangement mental, ne parvient à s'exprimer qu'en chantant. et encore développe-t-il une langue imprécise et hésitante qui confère à son personnage un caractère mystérieux et parfois même inquiétant ${ }^{36}$. Lord Laroque de Luna, personnage à la fois distingué et fantasque, use quant à lui d'une langue qui participe d'une stratégie de provocation: il cherche à libérer les refoulements des autres personnages et pour cela s'exprime en une langue haute en couleur, faite de répétitions, d'approximations et d'un extraordinaire mélange d'idiomes. Il cherche en cela à faire céder les défenses de ses interlocuteurs et à mettre en lumière leurs secrets honteux; cette incessante provocation verbale sera efficace ${ }^{37}$.

Ce qui unie tous les personnages de Willems, c'est donc la certitude que la langue, les mots dissimulent autant qu'ils expriment la réalité. Tous peuvent prendre pour

35 A ce propos, cf. les commentaires d'Alberte Spinette, dans P. Willems, Elle disait [...], op. cit., p. $169-177$.

Notons encore que la situation des personnages sur scène ainsi que la construction des dialogues contribuent, à la mode willemsienne, à fonder un monde étonnant, une fois encore en margc: comme si Ies distances et le temps ćlaient abolis, on retrouve sur la scène le pèrc et la mère d'Hélée, chacun d'un côté, et au centre Hélée et le soldat. Tous se parlent dans de faux dialogues qui, s'ils en ont parfois l'apparence, ne peuvent se lire comme tels puisque les personnages ne sont pas réunis dans la réalité ct que la mère est peut-être même morte.

${ }^{36}$ Le Vélicouseur: Je t'ai... (Même jeu. Il crache des bribes de syllabes comme si les mots élaient des couteaux qui le blessaient à la gorge). Je t'ai ... t'ai ... je ... je t'ai ... ai ... ai ... ai ... Je t'ai ... ai ... ai ... ai ...

Mesdemoiselles (mère du Vélicouseur), l'encouragcant: Oui! Parle, parle! Dis que tu m'aimes (...).

Lc Vélicouseur: Maman je t'ai ...

Mesdemoiselles: Il parle! II a presque dit Maman je t'aime». Il parle! Il va parler enfin! (Au Capitaine.) J'altends ce moment depuis vingt-deux ans. (Tout à coup délivrée de tout chagrin. Douceur.) Il m'aime.

Le Vélicouseur, en un élan affreux et splendide ct saisi peu à peu par une sorte de furcur inspirée [...]: Je t'ai ... Oui Ma mân-ân-ân! án! ân! Oui je t'ai ... Je t'ai en ... ho! ho! Je t'ai en ho ... Je t'ai en horreur! (En horreur» est expulsé triomphalement. Silence.) / P. Willcms, la Vita /...l, op. cit., p. 181-182.

Le Vélicouseur: Ma-man-ce-n'est-pas-moi-qui-ai-fu-é-1Ia-ma-li-ssa-Ma-man-c'est-moi-qui-ai-tu-é Ha-ma-li-ssa.

P. Willcms, La Vita /.../, op. cil., p. 246.

${ }^{37}$ Lord Laroque de Luna: Dear Brackx. I'm sorry for him! Son avenir est obscurissime, catastrophissime même. Avrà delle seccature. / Brourilles!' Ma! (Geste à l'italienne.) A puce futée Gros benêt mordu. / Ma! (Même geste.) Quella insolenza, quel l'altessa! Quel vapore, quella locomotiva! Et ça un soir de bonheur!

P. Willems, La Vita [.../, op. cit., p. 215 et p. 217. 
leur la formule d'une des ses héroînes: «Si je savais ce qu’il y a sous les mots, je saurais mon avenir» ${ }^{38}$.

C'est aussi le dilemme de Paul Willems, l'un des derniers grands écrivains de la Flandre francophone: s'il s'efforce à connaître le sens caché des mots, c'est parce qu'il est tributeur de deux cultures, situé «entre deux langues» ${ }^{39}$.

Ainsi, «'ineffable» tant caractéristique d'Aquélone, désignerait la Belgique unie, ou plutôt la Flandre avant la séparation communautaire, si l'on songe à l'éthymologie du nom ${ }^{t 0}$, en même temps mettant l'accent sur l'impossibilité à dire, problème auquel se heurtent les écivains belges de langue française.

\section{BIBLIOGRAPHIE}

Berg Ch., D'un pavs nové: la littérature francophone contemporaine en Flandre, dans Septentrion. Revue de culture néerlandaise, $21 \mathrm{e}$ annéc, $\mathrm{n}^{0} 4,1992$, p. 49-52.

Entretiens entre Paul Emond et Paul Willems les 7 mars et 9 avril 1979, transcrits d'après bande magnétique (45 p.).

$\mathrm{K}$ l ink e nberg J.-M., La production littéraire en Belgique francophone. Esquisse d'une sociologie historique, dans Littérature $\mathrm{n}^{0}$ 44. L'institution littéraire II. 1981, p. 33-50.

Le Monde de Paul Willems. Textes, etudes, documents rassemblés par Paul Emond, Henri Ronse et Fabrice van de Kerckhove, Labor, Bruxelles 1984 (Coll. Archives du futur).

Quaghebcur M., Belgique. La première des littératures francophones non françaises, Luxcmbourg, Association des Professeurs de Français du Grand-Duché de Luxembourg. 1991 (Coll. Les Cahiers Pedagogiques).

Spinettc A., Commentaires dans Willems P., Elle disait /.../. p. 169-177.

Ste iner G., Après Babel Une poétique du dire et de la traduction, trad. de l'anglais par Lucienne Lotringer, Albin Michel, Paris 1978 (et plus particluierement le chapitre 111 intitulé Le mor contre l'objet).

Willems P.. Un arrière-pavs. Reveries sur la creation littéraire, Louvain-la-Neuve, Presses universitaires de Louvain UCL, 1989 (Coll. Chaire de poétique $n^{\circ} 3$ ).

Wille ms P., L'auteur dramatique flamand de langue française, dans Etudes de littérature franf̧aise de Belgique offertes à Joseph llanse pour son 75 e anniversaire, éd. Jacques Antoine, Bruxelles 1978, p. 31-34.

Willems P., La Chronique du Cygne, Plon. Paris 1949.

Wille ms P., Elle disait dormir pour mourir, le Rideau de Bruxelles, 3e éd., Bruxclles 1994 (Les Cahiers du Rideau $\mathrm{n}^{\circ} 16$ ).

Willems P., Le Marché des petites heures, Bruxelles 1983. p. $64-137$ (Coll. Textes pour Didascalies $\left.n^{\circ} 6\right)$.

${ }^{38}$ P. Will cms, La ville à voile, op. cit., p. 78.

39 A. Spinettc, lecture de Blessures de Paul Willems, Espace Nord, Bruxelles 1984, p. 179.

${ }^{40}$ Aquélone fait penser d'une part à aquilon, ce vent poćtique du Nord, d'autre part il rejoint le vocabulaire aquatique, prolongé dans l'appelation des filles d'eau et dans le rỏle que l'cau, en l'occurence le fleuve, joue dans la pièce. 
Willems P., Nuit avec ombres en couleurs, Bruxelles 1983, p. 7-63 (Coll. Textes pour Didascalies $n^{0} 6$ ).

Willems P., Le pays noyé, Fata Morgana, s. I, 1990, 85 p.

Willems P., Il pleut dans ma maison, le Rideau de Bruxclles, Bruxclles 1976 (Les Cahiers du Rideau $\mathrm{n}^{\mathrm{0}} 3$ ).

Willems P., A la recherche d'un langage, dans Marginales. Revue bimestrielle des idées et des lettres, $\mathrm{n}^{0}$ 112-113, mai 1967, p. 105-106.

Willems P.. La Ville à voile, Labor, Bruxelles 1989, p. 13-148 (Coll. Espace Nord $n^{0} 55$ ).

Willems P., La Vita breve, Labor, Bruxelles 1989, p. 147-257 (Coll. Espace Nord $\mathrm{n}^{0} 55$ ). 